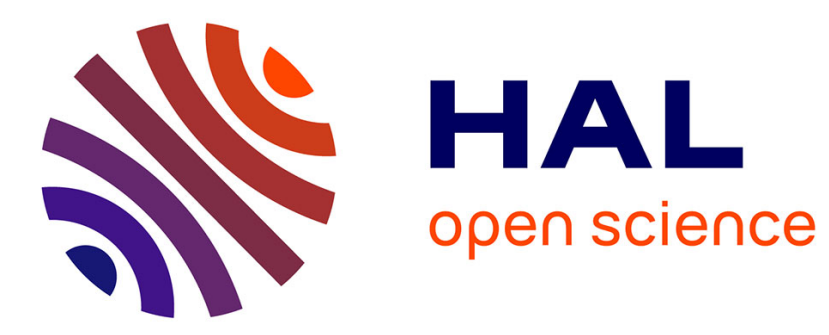

\title{
Le fait du loup. De la peur à la passion
}

Véronique Campion-Vincent, Jean-Claude Duclos, Christian Abry

\section{To cite this version:}

Véronique Campion-Vincent, Jean-Claude Duclos, Christian Abry. Le fait du loup. De la peur à la passion. Le monde alpin et rhodanien, 2002. halshs-01698200

\section{HAL Id: halshs-01698200 \\ https://shs.hal.science/halshs-01698200}

Submitted on 6 Feb 2018

HAL is a multi-disciplinary open access archive for the deposit and dissemination of scientific research documents, whether they are published or not. The documents may come from teaching and research institutions in France or abroad, or from public or private research centers.
L'archive ouverte pluridisciplinaire HAL, est destinée au dépôt et à la diffusion de documents scientifiques de niveau recherche, publiés ou non, émanant des établissements d'enseignement et de recherche français ou étrangers, des laboratoires publics ou privés. 


\section{Le fait du loup. \\ De la peur à la passion...}

Véronique Campion-Vincent, Jean-Claude Duclos et Christian Abry,

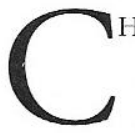

HACUN le sait, le loup est réapparu en France au début des années 1990, suscitant au fil des ans un ample débat de société. Révélée par des positions souvent passionnées, cette question mérite bien une réflexion de fond. Aussi l'équipe de rédaction du Monde alpin et rhodanien 'a-t-elle souhaité, à la suite de la table ronde organisée en 1999 par le Musée dauphinois (cf. la revue L'Alpe $\mathrm{n}^{\circ} 3$, mai-juillet 1999), procéder à un état des lieux des connaissances dont elle pouvait disposer, autant qu'à leur examen critique. Il en résulte ce numéro et, à la clef, une grave question: entre ceux pour qui la nature n'est naturelle qu'en l'absence de l'homme et qui se réjouissent du retour du loup, et d'autres qui ne peuvent concevoir de nature sans l'homme, qui l'emportera? À chacun, bien évidemment, de forger son opinion et de se demander si la possibilité d'une solution médiane est réaliste ou non. Nous n'omettrons pas cependant de constater que les citadins que la présence du loup enthousiasme n’en éprouvent aucune contrainte, tandis que beaucoup d'éleveurs et de bergers commencent à se demander si cette nouvelle présence animale n'exigera pas leur sacrifice. Certes, les dégâts provoqués par les attaques du loup sont indemnisées et d'importantes contributions financières sont consenties par la Commission Européenne pour tenter de faire cohabiter les troupeaux et le loup. Mais que répondraient, à la promesse d'une indemnité, un chercheur dont un pirate informatique détruirait le disque dur contenant ses travaux les plus récents ou un archéologue qui retrouverait sa fouille saccagée? Que diraient-ils, en effet, s'il fallait de plus qu'ils s'astreignent à vivre dans la hantise de la récidive?

Si une majorité de l'opinion se prononçait en faveur du loup et d'une nature d'autant plus belle et naturelle que l'homme en serait absent, alors faudra-t-il assumer aussi la disparition progressive du pastoralisme alpin et, entre autre, de la 
pratique multiséculaire de la grande transhumance ovine. Tel est en substance l'avis des éleveurs et des experts qui étudient leur activité.

En attendant, faisons le point. Quelles observations suscitent les faits contemporains et que livrent, sans a priori aucun, et principalement dans la région des Alpes et du Rhône, les données objectives de l'histoire?

La première partie de ce numéro tente de comprendre comment l'image du loup s'est transformée sous l'influence de mouvements de protecteurs et d'amis des loups, pour passer de celle d'animal absolument dangereux - dont la prédation sur l'humain et tout spécialement l'enfant, était considérée comme absolument intolérable - à celle d'animal parfaitement inoffensif pour l'homme, et d'emblème d'une nature reconstituée. Véronique Campion-Vincent détaille ainsi l'évolution de la situation de 1993 à 2002, un Plan Loup de régulation de l'espèce n'ayant été adopté qu’en 2000; elle examine les parties en présence - éleveurs et représentants politiques des zones montagnardes, associations de protecteurs et mouvements d'amis des loups, administrations - et leurs argumentaires - qui tous se réclament de «la nature». L'évolution de l'image de l'animal, du rejet à l'emblème de la reconstitution de la nature, est ensuite étudiée et ses liens avec des lâchers liés à l'existence de nombreux loups captifs soulignés. Situant la polémique autour des causes du retour des loups dans l'ensemble des rumeurs de lâchers d'animaux (dont les lâchers de vipères et de félins-mystère), Véronique Campion-Vincent propose de prendre au sérieux ces rumeurs, discours méprisés mais persistants qui montrent que les mesures de protection de prédateurs «ne vont pas de soi».

Analysant les représentations contemporaines du loup chez les éthologues, Sophie Bobbé souligne qu'elles visent sa réhabilitation, en légitimant ses prédations et en niant à peu près unanimement que le loup puisse être dangereux pour l'homme. Ces représentations sont mythiques et s'appuient (tout en le niant) sur le motif du loup dévorateur, bien qu’elles se prétendent scientifiques. À l'arrivée des loups en France, ces représentations ont nourri le discours médiatique, qui s'est focalisé sur la seule prédation lupine, ignorant les prédations de chiens divagants sur les troupeaux.

Sergio Dalla Bernardina passe en revue les productions les plus diverses de la culture de masse contemporaine - des livres pour enfants aux documentaires animaliers - dans lesquelles on s’identifie au loup réhabilité. Au terme de ce parcours qui fera souvent sourire, il analyse les motivations inexprimées et contradictoires de l'engouement actuel pour le loup: fascination face à sa force et à sa violence, mais aussi retour du réenchantement du monde par le recours au fantastique, à l'émotionnel et au sacré. La négation de la violence fascinante du loup permet projections fantasmatiques et expériences voyeuristes: ce n'est pas être sadique que d'assister à la dévoration des proies du prédateur «naturel».

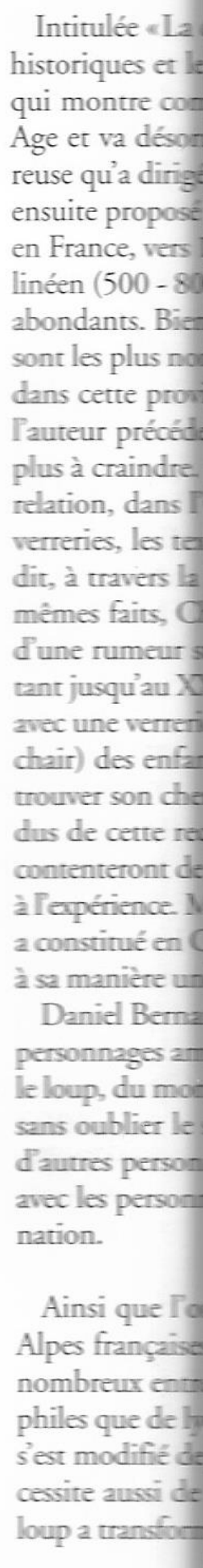


Intitulée "La crainte du loup», la deuxième partie du numéro aborde les faits storiques et les croyances. Elle commence, avec l'article de Gherardo Ortalli montre comment l'image négative du loup s'est précisée au Haut Moyenet va désormais, des siècles durant, inspirer la peur. Avec l'enquête rigouse qu’a dirigée Alain Molinier à la fin des années 1980, c’est aux faits qu’il est suite proposé de revenir. Ainsi apprend-on qu'il y a presque partout des loups France, vers 1800 , entre 100 et $1500 \mathrm{~m}$ d'altitude mais que c'est à l'étage colhéen $(500-800 \mathrm{~m})$, celui de la chênaie-charmaie précise-t-il, qu'ils sont les plus ondants. Bien que le Dauphiné n'apparaissent pas parmi les zones où les loup t les plus nombreux, c'est cependant par milliers que René Favier dénombre, tans cette province, ceux dont la monarchie rémunère l'élimination. Comme auteur précédent, il note que c'est dans les reliefs boisés que la menace est la plus à craindre. C'est d'ailleurs ce qu'illustre Raymond Moyroud en mettant en elation, dans l'une de ces forêts du Bas-Dauphiné où s'étaient implantées des erreries, les textes relatifs aux décès d'enfants causés par des loups et ce qu'en it, à travers la figure du loup-garou, la tradition orale. Prenant appui sur ces nêmes faits, Christian Abry et Alice Joisten conduisent leur enquête à propos dune rumeur structurelle observée, là, dès le milieu du XVIII siècle et persisant jusqu’au XXe, suivant laquelle "seigneurs ou ecclésiastiques, ayant partie liée vec une verrerie, envoient des loups-garous, leurs sbires, récupérer la graisse (ou chair) des enfants, pour la fabrication du verre». Laissant au lecteur le soin de trouver son chemin dans les fondements et les prolongements souvents inattendus de cette recherche qui l'entraînera jusqu'en Amérique du Sud, nous nous contenteront de signaler son objectif, soit de voir quels liens unissent les croyances l'expérience. Montrant comment «le légendaire» du loup, à partir du corpus qu'il constitué en Cévennes, s'inscrit dans l'espace et le temps, Pierre Laurence tente sa manière une quête comparable.

Daniel Bernard, avec qui s’achève cette partie, décrit quant à lui la gamme des personnages ambigus qui, dans la société traditionnelle, maitrisent et contrôlent - loup, du montreur au meneur dont les pratiques sont proches de la sorcellerie, ans oublier le saint qui transforme l'animal en auxiliaire soumis. Aujourd'hui 'autres personnages fascinent par leur proximité à l'animal et la comparaison vec les personnages d'hier est tentante: il s'agit toujours de charme et de fascination.

Ainsi que l'on pouvait s'y attendre, c'est aux Alpes et plus précisément aux Alpes françaises, que s'intéressent les auteurs de la dernière partie. À partir de nombreux entretiens effectués dans le massif de la Vanoise, tant auprès de lycophiles que de lycophobes, Isabelle Mauz montre comment le rapport au sauvage sest modifié depuis la réapparition du loup; et comment traiter du sauvage nécessite aussi de traiter de l'humain. Ainsi observe-t-elle comment le retour du loup a transformé les rapports des hommes entre eux. Ce sont ensuite aux éleveurs 
des quatre grands systèmes pastoraux exposés au loup - montagnard, préalpin, transhumant et herbassier - que Patrick Fabre et Guillaume Lebaudy s'intéressent. Analysant les perturbations provoquées par les loups dans l'accomplissement du métier pastoral, ces deux chercheurs vont jusqu'à se demander s'il n'y a pas là les stigmates d'une divergence pour le moment irréconciliable. Marc Mallen, quant à lui, étudie les réactions des bergers, soit des salariés des éleveurs. Obligés à prendre de nouvelles dispositions, ces bergers voient, dans la menace du loup, l'opportunité de faire valoir et reconnaître un métier en voie de dévalorisation.

Avec la dernière contribution de ce numéro, Laurent Garde procède à l'inventaire et au «démontage» méthodique de chacune des positions émises, tant par les protecteurs du loup que par ses détracteurs. Ainsi parvient-il à remettre en cause l'argument si souvent opposé par ceux qui ont à cœur de disculper le loup en dénonçant les méfaits des chiens errants. Comme Isabelle Mauz, Patrick Fabre et Guillaume Lebaudy, Laurent Garde explique comment le loup radicalise les positions quant aux représentations de l'espace naturel. À ceux qui restent du monde rural et ne peuvent concevoir cet espace que profondément façonné par l'homme, s'opposent en effet de manière répétéeles partisans d'une nature indépendante de l'homme, indemne de sa présence et vierge.

Telles sont les pièces qui constituent, comme celles d'un procès d'instruction, le dossier de ce numéro. Autant d'articles et d'approches, de l'hier à l'aujourd'hui pour contribuer à «faire juges de leur propre cause» ceux qui trouveront là, sinon de nouveaux éclairages, d'autres questionnements. Le retour du loup aura au moins le mérite de stimuler notre réflexion sur la nature humaine. Puisse-t-elle, tandis que l'Assemblée nationale crée, en novembre 2002, une commission intitulée "Conditions de la présence du loup en France et de l'exercisme du pastoralisme dans les zones de montagne», contribuer au débat.

Véronique CAMPION-

VINCENT

CNRS, Maison des Sciences

de l'Homme, Paris
Jean-Claude Duclos

Musée Dauphinois, Grenoble
Christian ABRY

CARE et

université Stendhal

Grenoble 